\title{
Thyristor Compact Modeling based on Gummel-Poon Model Including Parameter Extraction Procedure
}

\author{
Ahmed Shaker \\ Lecturer of Engineering \\ Physics \\ Faculty of Engineering \\ Ain Shams University \\ Cairo, Egypt
}

\author{
Gihan T. Sayah \\ Lecturer of Electronic \\ Engineering \\ Nuclear Materials \\ Authority \\ Cairo, Egypt
}

\author{
Mohamed Abouelatta \\ Lecturer of Electronic \\ Engineering \\ Faculty of Engineering \\ Ain Shams University \\ Cairo, Egypt
}

\author{
Abdelhalim Zekry \\ Professor of Electronic \\ Engineering \\ Faculty of Engineering \\ Ain Shams University \\ Cairo, Egypt
}

\begin{abstract}
In this paper, an improved approach for the modeling of power thyristors is presented. A modified two-transistor configuration based on the Gummel-Poon model is applied. This model takes into account the conductivity modulation and carrier-carrier scattering by using nonlinear current sources. The current gain of the transistor is studied relating it to the injection level in order to provide a more insight of some SPICE parameters. The design parameters of the thyristor are related to circuit parameters using some analytical expressions. Then, the SPICE model parameters are extracted using Silvaco. The simulation results are compared with measurements showing good agreement indicating that the developed model could efficiently describe the performance of the thyristor under various practical operating conditions.
\end{abstract}

\section{General Terms}

Thyristor Modeling, TCAD, PSPICE.

\section{Keywords}

Modeling, Gummel-Poon, Conductivity Modulation, Design Parameters, Parameter extraction.

\section{INTRODUCTION}

Several thyristor models have been developed for use in circuit simulators. Some models were developed by solving the semiconductor equations numerically. Ma and Lauritzen [1] developed a physical SCR (Silicon Controlled Rectifier) model by employing the Lumped-Charge modeling technique to simplify the device physics. The model parameters were few and didn't describe the performance of the device completely. A model based on the Fourier-series approach was implemented for system simulation using Matlab [2]. This approach was first proposed by Kallala [3] whose model was implemented in ESACAP [4].

Other models were based on the two-transistor configuration assuming Ebers-Moll model. Williams [5] developed a dc and ac model by applying the Ebers-Moll BJT model to the twotransistor configuration. Because relatively few model parameters can be extracted from measured data, this model is not proper for simulation purposes. Brambilla et al. [6] developed a numerical SCR model based on the Ebers-Moll equations and a topology represented by the three-junction devices. The parameter extraction of these models was not provided which limits the applicability of these models.

\begin{abstract}
The two-transistor model based on Gummel-Poon BJT model was a motivation for many published papers. The first who considered that modeling technique was $\mathrm{Hu}$ and $\mathrm{Ki}$ [7]. Then, a composite model for the GTO (Gate Turn OFF) was introduced by Xiangning et al. [8]. A parametric sensitive analysis was performed with the complete composite model, and comparison between experiment and SPICE simulations was provided. Good results of this model indicate the validity of the model and the superiority to use the Gummel-Poon model instead of the Ebers-Moll model.
\end{abstract}

Recently, Sayah et al. [9] proposed a SPICE model based also on the Gummel-Poon model and taking conductivity modulation into account. The advantage of this work is that a full parameter extraction sequence is provided [10]. This modeling technique is chosen in this paper as a starting point for the model. Some effects are added by modeling the conductivity modulation of the base region including the carrier-carrier scattering which is important to accurately adjust the static characteristics. Also, a detailed study is provided to demonstarte the variation of the current gains of the two transistor components of the model with the injection level in order to provide an accurate identification of the SPICE parameters of the model. An extraction of the design parameters is provided based on some analytical expressions and a trimming of these parameters is done using measurements. Finally, a case study is taken and its design parameters are extracted. Static and dynamic behaviors using SPICE simulations are compared with measurements showing good agreement.

\section{MODEL DESCRIPTION}

\subsection{Main Structure}

The basic structure of the thyristor is shown in Fig. 1(a) which also indicates the emitter short appearing as a simple resistance $R_{s h}$. The resistance of the lightly doped n-base layer of the thyristor to the anode current flow is modeled by the resistance $R_{n b}$. The complete model is represented in Fig. 1(b) where the two transistors are modeled using Gummel-Poon equivalent circuits. Actually, the two transistor model could be used very efficiently in modeling the thyristor and other relating devices. But it needs some modifications to accurately predict the terminal characteristics both static and dynamic. 


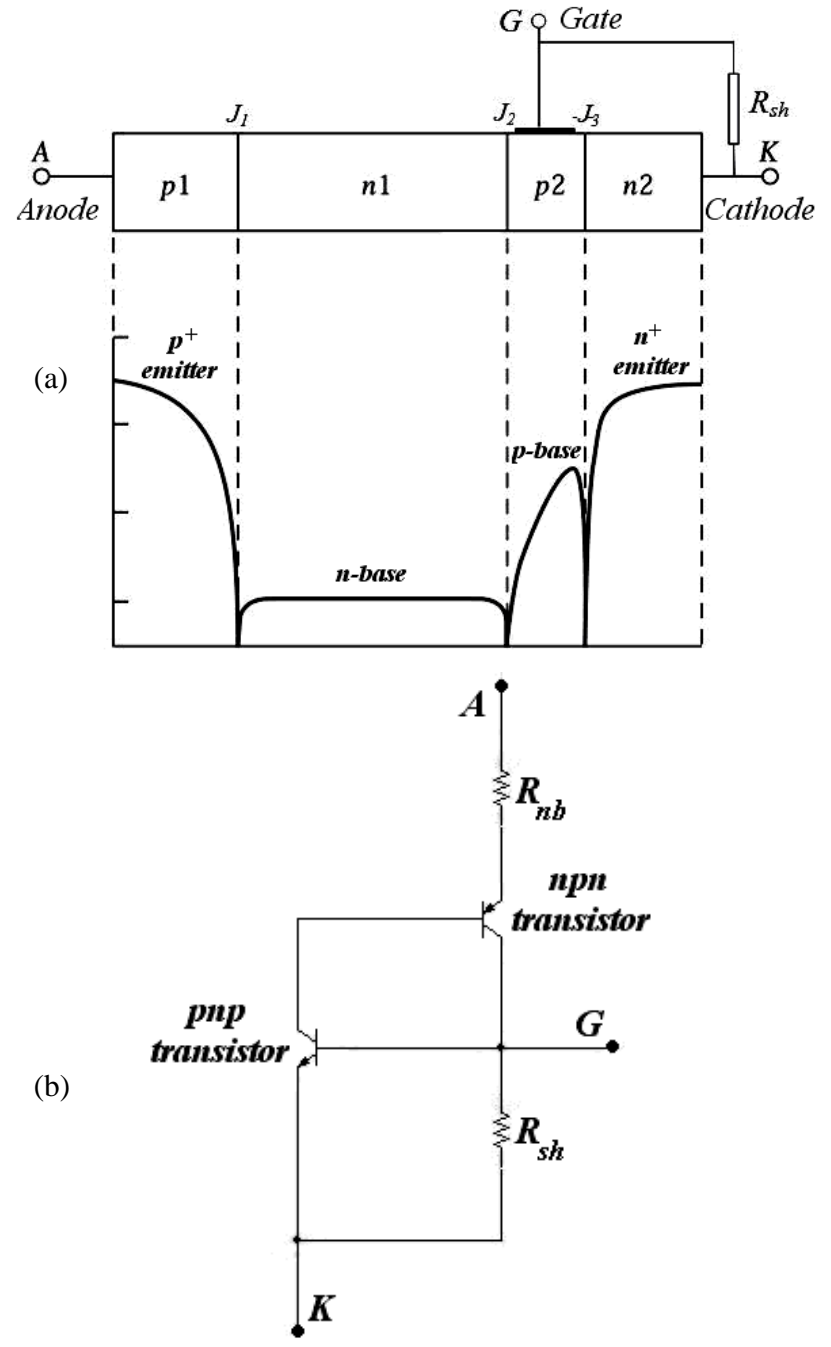

Fig 1 (a) Basic structure of the thyristor and (b) The twotransistor SPICE model

\subsection{Conductivity Modulation and Carrier- Carrier Scattering}

Concerning the resistance of the low-doped $n$-base, it could be modeled as follows:

$$
R_{n b}=\frac{W_{n B}}{q A\left[\left(\mu_{n}+\mu_{p}\right) \Delta p+\mu_{n} N_{D B}\right]}
$$

where $\Delta p$ is the average excess hole concentration in the $n$ base. The sum of mobilities could be expressed in terms of the average excess hole concentration as [11]:

$$
\mu_{n}+\mu_{p}=\frac{n_{o} \mu_{o}}{\Delta p+n_{o}}
$$

Then inserting Eq. 2 into Eq. 1 leads to:

$$
\frac{1}{R_{n b}}=\frac{q A \mu_{n} N_{D B}}{W_{n B}}+\frac{q A \mu_{o} n_{o} \Delta p}{W_{n B}\left(\Delta p+n_{o}\right)}
$$

It could be deduced from the previous equation that the n-base resistance could be expressed in terms of three basic resistances; $R_{\text {onb }}, R_{\text {lim }}$, and $R_{\text {mod }}$. $R_{\text {onb }}$ is the unmodulated base resistance, $R_{\text {lim }}$ is a limiting resistance resulting from carriercarrier scattering, and $R_{\text {mod }}$ is resistance resulting directly from the excess hole concentration. In conclusion, the n-base total resistance including both effects of conductivity modulation and carrier-carrier scattering could be expressed as follows:

$$
R_{n b}=R_{\text {onb }} / /\left(R_{\text {lim }}+R_{\text {mod }}\right)
$$

where

$$
\begin{aligned}
& R_{\text {onb }}=\frac{W_{n B}}{q A \mu_{n} N_{D B}} \\
& R_{\text {lim }}=\frac{W_{n B}}{q A \mu_{o} n_{o}} \\
& R_{\text {mod }}=\frac{W_{n B}}{q A \mu_{o} \Delta p}
\end{aligned}
$$

The excess hole concentration can be approximately expressed in terms of the anode current as [12]:

$$
I_{A}=\frac{q A\left(W_{n B}+W_{p B}\right) \Delta p}{\tau_{p}}
$$

Finally the voltage drop on the conductivity modulated n-base is modeled as:

$$
V_{n B}=I_{A} R_{n b}
$$

It is emphasized that the resistance $R_{n b}$ accounts for the total resistor effect of the n-base which is the base region of the pnp transistor, so $R_{n b}=0$ is used for the SPICE pnp BJT component in the simulation.

\section{NPN AND PNP CURRENT GAINS}

One of main issues that should be covered well is the study of the DC current gains (dc alphas) and the small-signal current gains (ac alphas). These alphas play an important role in the modeling of the thyristor considering the two-transistor model. Although many contributions are found in literature considering the measurements and simulation of various thyristor structures [13], the phenomenon of alpha behavior according to the injection level is not covered well.

It is important to study this effect because the proposed model depends on some parameters which is very important to be modeled well or the results will deviate noticeably from measurements. Some of the vital model parameters are the SPICE parameters $I_{K F}$ and $I_{K R}$. These parameters could be determined falsely leading to simulation errors. When injection levels are not taken into consideration, there will be errors in evaluating the current gains that couldn't be ignored.

To study this effect in details, a thyristor structure whose fabrication details are given in [14] and [15] is simulated. A process simulator Athena Silvaco [16] is used for simulation of the fabrication processes and a device simulator Atlas Silvaco [17] is used for simulation of the terminal characteristics. First the thyristor is decomposed of its two constitute two transistors. Each transistor is virtually fabricated and simulated separately. Then the alphas of the each component are determined. The comparison with measurements is done whenever possible. 


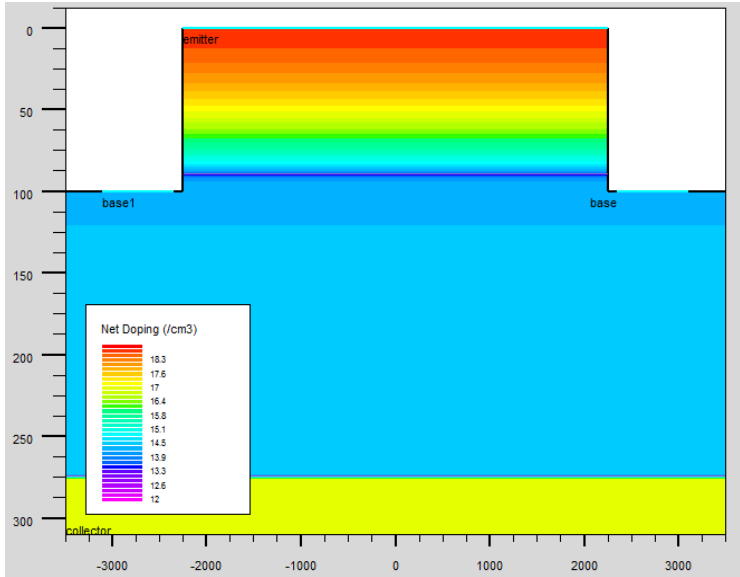

(a)

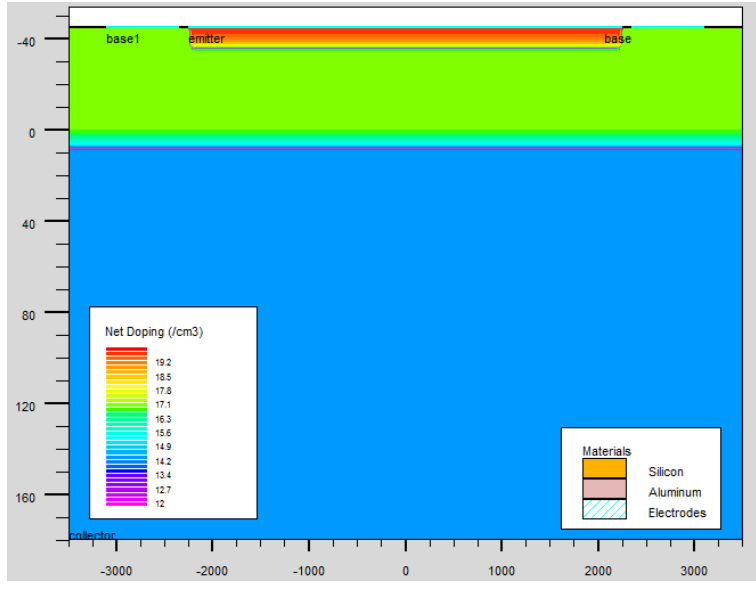

(b)

Fig 2 Transistors components of the thyristor: (a) pnp and (b) npn

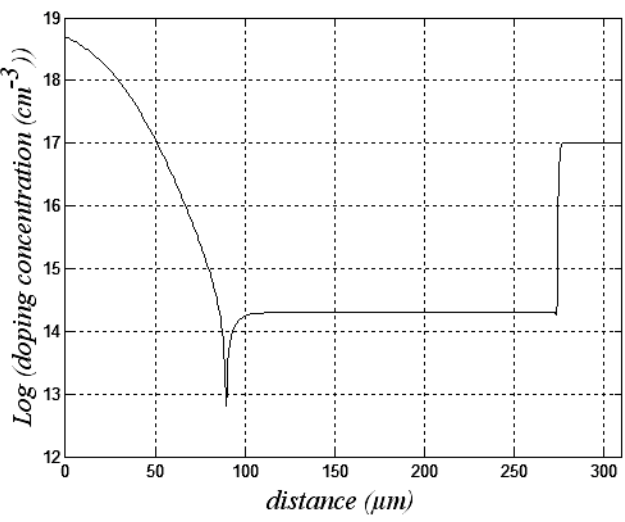

(a)

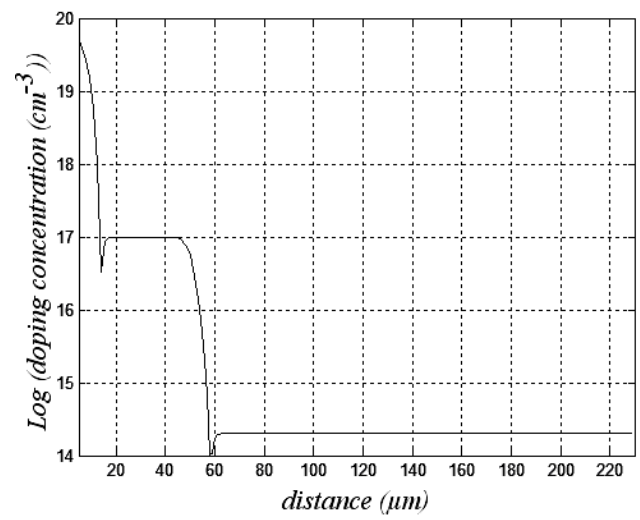

(b)

Fig 3 Doping profiles of the thyristor components: (a) pnp and (b) npn

The simulated thyristor structure is presented in [14]. The nbase width is about $180 \mu \mathrm{m}$ with doping of $2 \times 10^{14} \mathrm{~cm}^{-3}$. The p-base is an epitaxial layer whose doping is ranging from $1 \times 10^{16} \mathrm{~cm}^{-3}$ to about $1 \times 10^{18} \mathrm{~cm}^{-3}$. Now considering this thyristor structure and dividing it to its two transistor equivalence following the measurement technique by Gerlach [15] as shown in Fig. 2. The corresponding doping profiles are shown in Fig. 3.

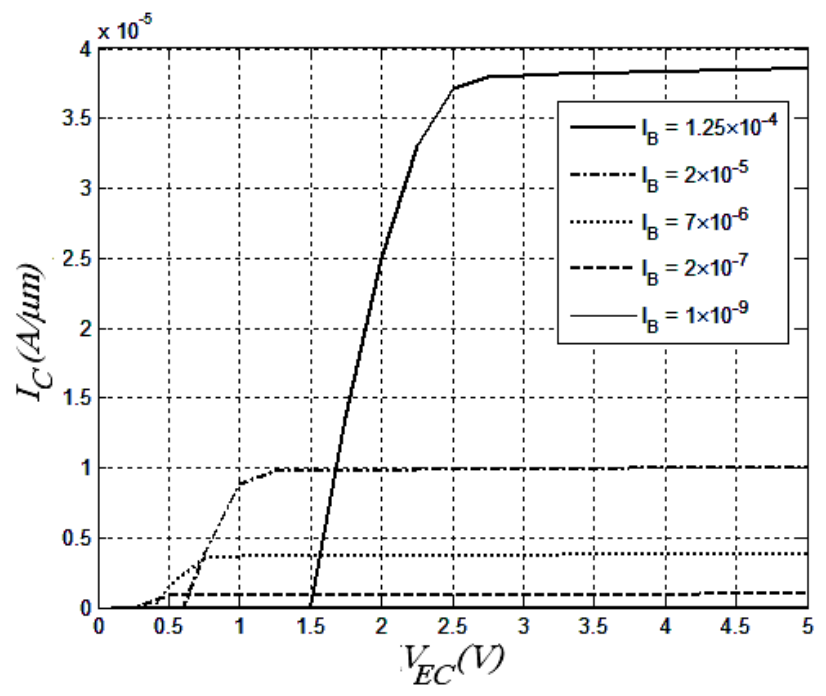

Fig 4 The transfer characteristics of the pnp; all currents are given per width
In order to obtain the alphas of each transistor, DC simulations are carried out, using Silvaco, in which the base current is varied in fine steps (such as to prevent simulation convergence) for different values of $V_{C E}$. A family of $I_{C}-V_{C E}$ curves results. For instance, in the case of the pnp transistor, Fig. 4 shows these family of curves. All important physical semiconductor parameters are taken into account like the carrier-carrier scattering, band gap narrowing, and dopingdependent lifetime and mobility. Using the resulting $I-V$ characteristics, the alphas are easily extracted.

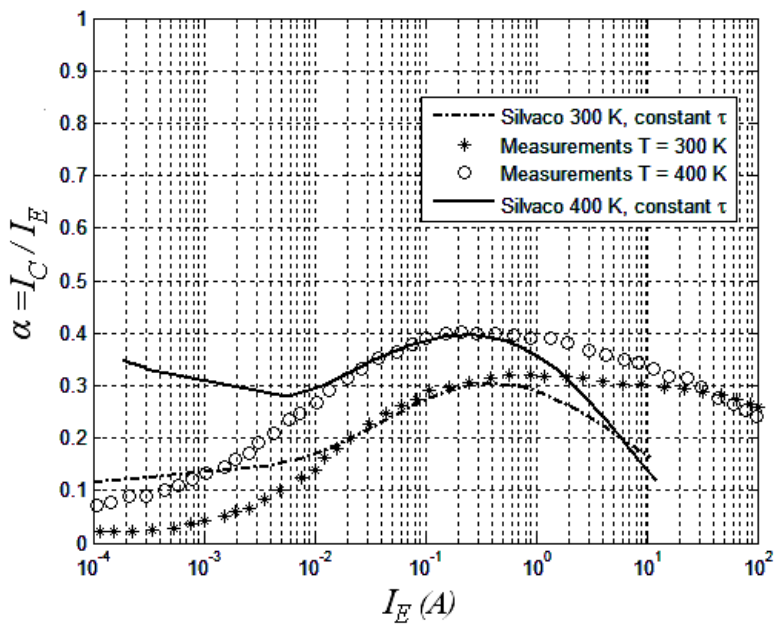

Fig 5 DC current gain of the pnp considering constant lifetime 
Fig. 5 shows the corresponding alpha of the pnp transistor for a lifetime of $\tau_{H L}=10 \mu \mathrm{s}$. As can be deduced from the figure, there are disagreements between the simulation and measurements. This is mainly accounted for by the constant lifetime model taken in the simulation. The disagreement increases at low level injection for high temperature because of increasing of lifetime with temperature.

The discrepancy between simulation and measurements could be reduced if the variation of the lifetime with injection level is taken. So, the lifetime is varied with the emitter current $I_{E}$. To reach the best fit with the measured data, the high-level lifetime is varied starting by $5 \mu$ s at low injection, reaching 8 $\mu \mathrm{s}$ to $10 \mu \mathrm{s}$ at medium injection, and ending with $15 \mu \mathrm{s}$ at high injection. As expected, the agreement becomes evident between measurements and simulations as shown in Fig. 6. To reach a good fit between simulation and measurements, a behavioral model is applied for the injection-dependent lifetime. Unfortunately, there is no such model in Silvaco. So, the lifetime is changed manually by incrementing it with current.

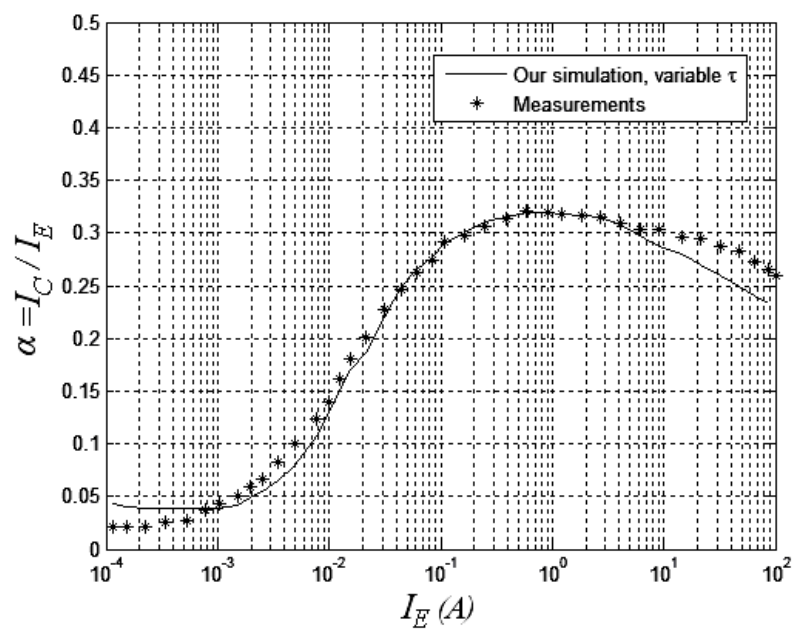

Fig 6 The DC current gain of the pnp considering variable lifetime

The conclusion is that the value of $I_{K F}$ expected by regular simulation is far from reality. The actual value is considerably larger than the value obtained by direct extraction. The problem when using a small value of $I_{K F}$ is that the summation of alphas may be below unity. In such case, the thyristor will be OFF while it should be ON. So, in the presented circuit model of the thyristor, $I_{K F}$ is chosen to take a higher value than extracted.

\section{IDENTIFICATION OF DESIGN PARAMETERS}

First a quick review of the main design and circuit parameters of the thyristor model used is provided. Then the relations between these parameters are provided. The main design and technological parameters for the power thyristor are:
1) Active die area, $A$
2) n-base width, $W_{n B}$
3) n-base concentration, $N_{D B}$ 4) Effective minority-carrier, and high-level lifetimes in the
wide n-base, $\tau_{p}$, and $\tau_{H L}$
5) p-base width, $W_{p B}$
6) p-base concentration, $N_{A B}$

The parameter extraction approach relies on how to relate these physical parameters to the circuit parameters. The main circuit and device parameters are:

1) Breakdown voltage between A-K (this is related to $V_{R R M}$ as in the case of power diodes), $V_{B R(\mathrm{AK})}$

2) Breakdown voltage between $\mathrm{G}-\mathrm{K}, V_{B R(\mathrm{GK})}$

3) DC forward current, $I_{F}$

4) The fall time, $t_{f}$

5) Device off time, $t_{q}$,

6) Forward voltage drop, $V_{F}$.

\subsection{Active Die Area}

An estimated value of the active die area $A$ can be obtained from the forward current $I_{F}$ (which should be indicated in the device datasheet). The current density $J$ for most power thyristors is ranging from $100 \mathrm{~A} / \mathrm{cm}^{2}$ to $500 \mathrm{~A} / \mathrm{cm}^{2}$. Then, similar to power diodes [18], the initial value of $A$ can be estimated as:

$$
A=\frac{I_{F}}{J}
$$

\subsection{Minority-Carrier Lifetime}

The minority-carrier lifetime in the n-base could be related approximately to either the turn-off time or the fall time of the thyristor. The turn-off time $t_{q}$ (which is usually found in datasheets) is related to $\tau_{p}$ via the following relation [19]:

$$
t_{q} \approx 4.6 \text { to } 6.9 \tau_{p}
$$

When the thyristor is commutated from a forward current $I_{F}$ to a reverse current $I_{R}$, the fall time $t_{f}$ of the reverse recovery current $I_{R}$ (which is the time required for $I_{R}$ to be reduced from $90 \%$ to $10 \%$ of its initial value at the storage delay time) is also directly related to the lifetime $\tau_{p}$, and it is given by [12]:

$$
t_{f} \approx 2.3 \tau_{p}
$$

\section{3 n-base Width and Doping}

The actual breakdown voltage for the thyristor in the reverseblocking mode is governed by the open-base transistor (P1N1P2) breakdown phenomenon. The optimization of the n-base width and doping is studied such that to obtain a specific value for the breakdown $V_{R}$ (which is also called $\left.V_{B R(\mathrm{AK})}\right)$. Following the approach given by Baliga [20], $V_{R}$ is varied for some selected different values of $\tau_{p}(=1,5$, and 10 $\mu \mathrm{s})$. The optimum base width and doping theoretically calculated are shown in Fig. 7 for different values of $V_{R}$.

To validate this theoretical approach, a typical thyristor structure is examined whose profile is shown in Fig. 8(a) with a wide $n$-base width of $360 \mu \mathrm{m}$ and $n$-base doping of $2.1 \times 10^{13} \mathrm{~cm}^{-3}$ [20]. The $\mathrm{n}$-base doping is varied from $1 \times 10^{13}$ to $7 \times 10^{13} \mathrm{~cm}^{-3}$ and the breakdown voltage is calculated for each case. The lifetimes are fixed at $\tau_{n}=\tau_{p}=3 \mu \mathrm{s}$. The comparison between the analytical model with Silvaco simulations is shown in Fig. 8(b). This comparison indicates good agreement and proves the usefulness of the analytical model used. 


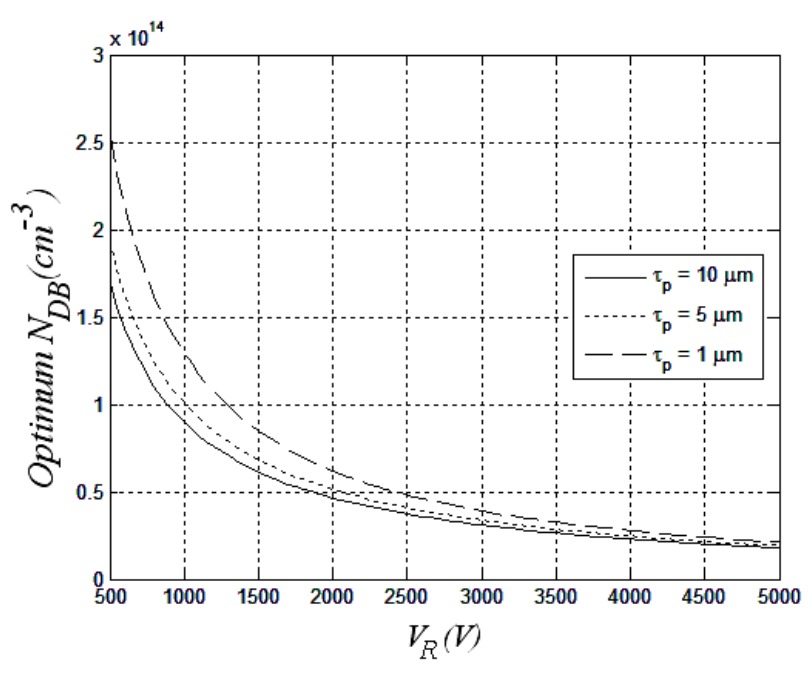

(a)

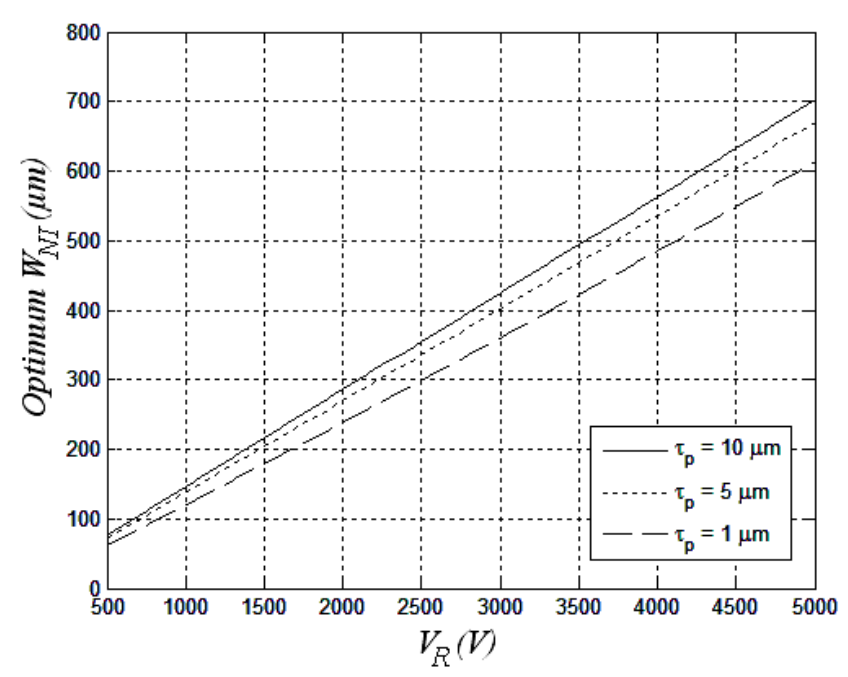

(b)

Fig 7 Optimum base width and base doping for $\tau_{p}=1,5$, and $10 \mu \mathrm{s}$

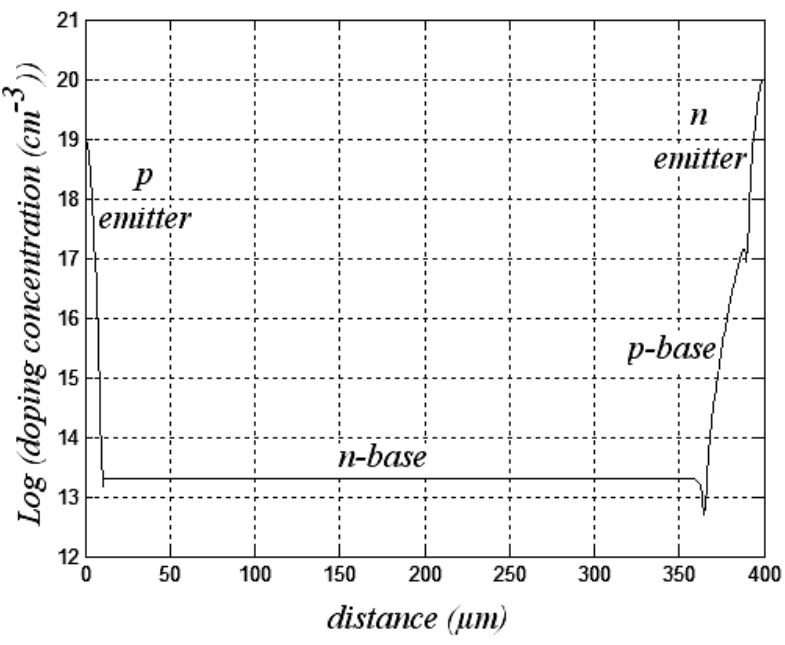

(a)

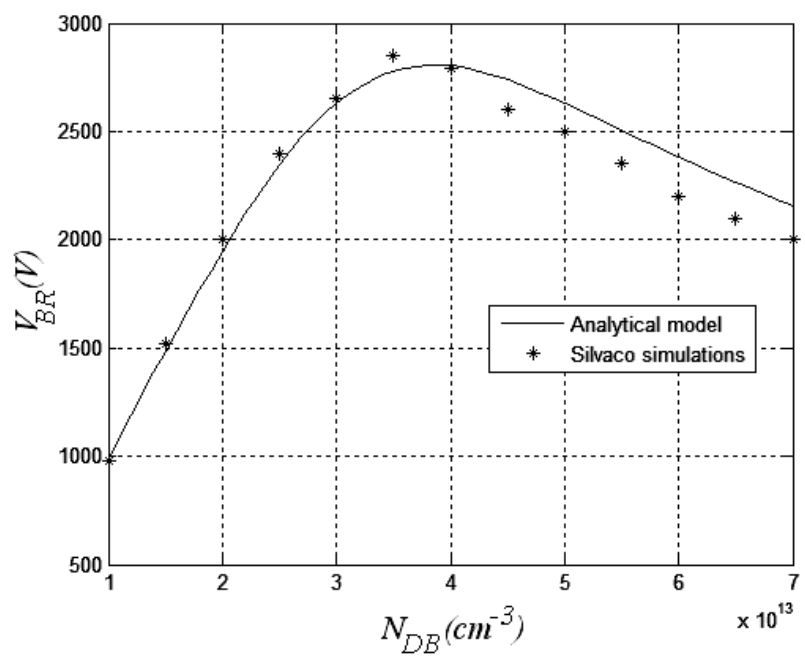

(b)

Fig 8 (a) Doping profile of a thyristor, (b) Comparison between analytical model and Silvaco simulations for breakdown calculations

\section{4 p-base Width and Doping}

The p-base doping could be related to the breakdown voltage between the gate and cathode $V_{B R(\mathrm{GK})}$. A simple analytical expression is used considering the critical electrical field concept:

$$
V_{B}=\frac{\varepsilon_{r} \varepsilon_{o} E_{M a x}^{2}}{2 q N_{A B}}
$$

where $E_{\operatorname{Max}}$ is the maximum electric field in the junction which is given by [21]:

$$
E_{\text {Max }}=4010 N_{B}^{1 / 8}
$$

Then, knowing $V_{B R}$ of the gate-to-cathode junction, Eq. 13 and Eq. 14 are solved to find the p-base doping $N_{A B}$. This doping should practically in the range of $1 \times 10^{16}-1 \times 10^{17} \mathrm{~cm}^{-3}$ to obtain a reasonable gain for the internal npn BJT component of the thyristor [20].

The base width $W_{p B}$ (or equivalently $W_{P 2}$ ) may be found based upon the constraint about the forward voltage drop or the average ON-state voltage. The method relies on assuming a PIN diode behavior for the thyristor and calculating the forward characteristics [22]. This is practically accepted for cases when high injection prevails. In this case, the equivalent base diode width is $W_{N 1}+W_{P 2}$ (where $W_{N 1}$ is the N-base width).

\section{SIMULATION AND RESULTS}

The BT151 is taken as a study case to validate the proposed model. Some selected design specifications from its datasheet are shown in Table 1. The main DC constraints are repetitive maximum voltage $V_{R R M}$, the peak reverse gate voltage $V_{R G M}$, and the forward current $I_{F}$. The main dynamic constraint is the turn-off time $t_{q}$ at a certain condition. 
Table 1 Main design specifications of BT151 thyristor

\begin{tabular}{|c|c|}
\hline$V_{R R M}$ & $800 \mathrm{~V}$ \\
\hline$V_{R G M}$ & $5 \mathrm{~V}$ \\
\hline$I_{F}$ (average) & $7.5 \mathrm{~A}$ \\
\hline$V_{F}$ (typical) at $I_{F}=23 \mathrm{~A}$ & $1.4 \mathrm{~V}$ \\
\hline$t_{q}$ (maximum) at $d i_{F} / d t=30 \mu \mathrm{A} / \mathrm{s}$ and $125^{\circ} \mathrm{C}$ & $70 \mu \mathrm{s}$ \\
\hline
\end{tabular}

\subsection{Evaluation of Design Parameters}

Following the methodology presented in the previous section, a try to extract the design parameters of this Thyristor is followed. The die active area could be found by assuming a forward current density of $200 \mathrm{~A} / \mathrm{cm}^{2}$ as an average value for a small thyristor. This gives an area of $A=0.0375 \mathrm{~cm}^{2}$.

To extract the minority-carrier lifetime $\tau_{p}$, the value of $t_{q}$ specified in the datasheet is used. From Eq. 11, a maximum possible $\tau_{p}$ is required. Noting that $t_{q}$ is given at a temperature of $125{ }^{\circ} \mathrm{C}$, the value of $\tau_{p}=15.2 \mu$ s obtained should be corrected to get it at room temperature. Using the temperature dependence of the lifetime given in [23], $\tau_{p}$ could be calculated at room temperature which is about $10 \mu \mathrm{s}$.

Next, consider the breakdown voltage from anode-to-cathode. Assuming a margin of about $400 \mathrm{~V}(50 \%$ safety factor is assumed), then the breakdown voltage $V_{B R(\mathrm{AK})} \approx 1200 \mathrm{~V}$. From Fig. 7 the n-base width and doping are obtained. The values are $N_{D B}=7.6 \times 10^{13} \mathrm{~cm}^{-3}$ and $W_{n B}=175 \mu \mathrm{m}$. For the breakdown voltage from gate-to-cathode, consider the datasheet constraint $V_{R G M}$. Then $V_{B R(\mathrm{GK})} \approx 5 \mathrm{~V}$. Using Eq. 13 and Eq. 14: $N_{A B}=2.18 \times 10^{17} \mathrm{~cm}^{-3}$.

Now, for p-base width $W_{p B}$, consider the forward voltage drop at a certain condition as given in the datasheet. Applying a DC model of the power PIN diode [22], and taking $\tau_{H L} \approx 20 \mu$ s, a suitable $W_{p \mathrm{~B}}$ is found such that $V_{F}$ (forward voltage) doesn't exceed $1.4 \mathrm{~V}$ at a current of $23 \mathrm{~A}$. A value of $W_{p \mathrm{~B}} \approx 70 \mu \mathrm{m}$ was found from these calculations. To check that the values are all in the correct range, the DC $I-V$ characteristics are simulated and compared with that given in the datasheet. Fig. 9 shows the difference between simulation and datasheet typical values for temperature of $125^{\circ} \mathrm{C}$. A good match is seen, assuming $h$-parameters of $3 \times 10^{-14} \mathrm{~cm}^{4} / \mathrm{s}$ on the average which is a suitable typical value.

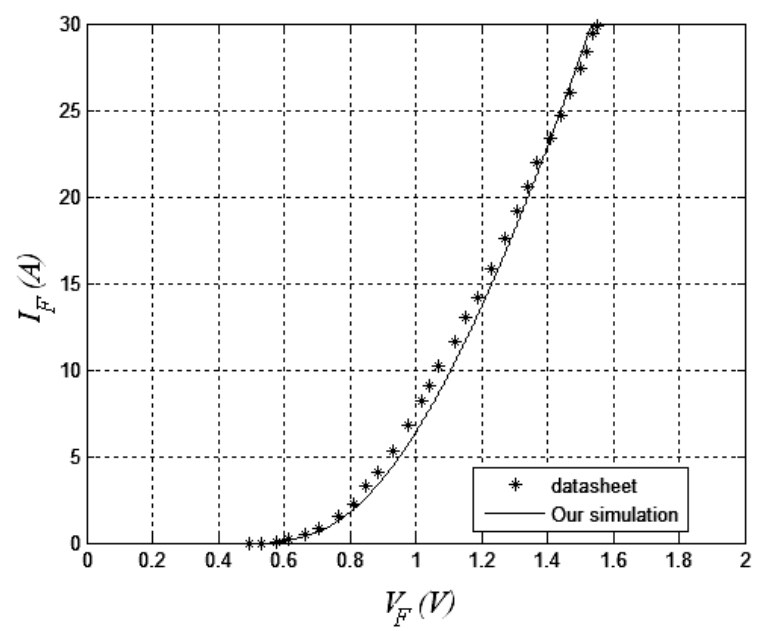

Fig 9 Comparison between simulation and datasheet DC characteristics

\subsection{Design Parameters Trimming Using Measurements}

First, the breakdown voltages were measured by a curve tracer and found to be $V_{B R(\mathrm{Ak})}=1200$, and $V_{B R(\mathrm{GK})}=5.2 \mathrm{~V}$. So, the doping levels and base widths will not be modified. The dynamic performance was measured and $t_{f}$ was found to be 24 $\mu \mathrm{s}$. Using Eq. 13, $\tau_{p}=10.4 \mu \mathrm{s}$ is calculated which is very close to the value obtained concerning $t_{q}$ constraint.

Next, a check for the area $A$ is done. $C-V$ measurements were taken between the gate-anode junction. It was shown that [10]:

$$
\frac{1}{C_{m}^{2}}=\frac{1}{C_{j_{o}}^{2}}\left[1+\left(1+\frac{V}{\varphi}\right)^{0.5}\right]^{2}
$$

where $C_{m}$ is the measured capacitance between the gate and the anode. $C_{j o}$ is the junction capacitance at zero bias which is given by:

$$
C_{j_{o}}=A \sqrt{\frac{q \varepsilon_{r} \varepsilon_{o} N_{D B}}{2 \varphi}}
$$

From capacitance measurement: $C_{j}=85.4 \mathrm{pF}$ and $\varphi=0.75 \mathrm{~V}$ Then the area $A$ could be obtained from Eq. $16: A=0.03 \mathrm{~cm}^{2}$ which is not far from the value obtained shortly. The difference may occur due to the uncertainty in determining the doping levels.

To complete the thyristor model one has to take into consideration the emitter shorts. A simplified model was proposed in [10]. From the model, the emitter short resistance was estimated to be $R_{s h} \approx 300 \Omega$. Table 2 shows a summary of the design parameters that that will be used in simulation.

Table 2 Design parameters used for simulations

\begin{tabular}{|l|c|c|}
\hline \multicolumn{1}{|c|}{ Physical parameter } & Symbol & Value \\
\hline $\begin{array}{l}\text { n-base doping concentration of } \\
\text { the pnp transistor }\end{array}$ & $N_{D B}$ & $7.6 \times 10^{13} \mathrm{~cm}^{-3}$ \\
\hline $\begin{array}{l}\text { p-base doping concentration of } \\
\text { the npn transistor }\end{array}$ & $N_{A B}$ & $2.18 \times 10^{17} \mathrm{~cm}^{-3}$ \\
\hline n-base width of pnp transistor & $W_{n B}$ & $175 \mu \mathrm{m}$ \\
\hline p- base width of npn transistor & $W_{p B}$ & $70 \mu \mathrm{m}$ \\
\hline Area of the junction & $A$ & $0.0375 \mathrm{~cm}^{2}$ \\
\hline Lifetime of minority carriers & $\tau_{p}$ & $10 \mu \mathrm{s}$ \\
\hline The emitter short resistance & $R_{S h}$ & $300 \Omega$ \\
\hline
\end{tabular}

Once these values for the technological parameters of the thyristor are extracted, Athena is used to fabricate virtually the two transistors. Then a built-in simulator in Silvaco called QUICKBIP is used to extract the Gummel-Poon SPICE parameters [24]. QUICKBIP is fully automated and takes all important bipolar semiconductor parameters into consideration. The extracted SPICE parameters for the pnp transistor and npn transistor are shown in Table 3. The values of $I K F$ and $I K R$ are not reliable for simulation as stated previously. Instead, suitable high values are used for SPICE simulation. 
Table 3 Extracted SPICE parameters for the pnp and npn transistors

\begin{tabular}{|l|c|c|c|}
\hline \multicolumn{1}{|c|}{ SPICE parameter } & Symbol & npn & pnp \\
\hline Saturation current & $I S$ & $5.6 \times 10^{-14} \mathrm{~A}$ & $1 \times 10^{-12} \mathrm{~A}$ \\
\hline Base-emitter saturation current & $I S E$ & $2 \times 10^{-14} \mathrm{~A}$ & $4.7 \times 10^{-11} \mathrm{~A}$ \\
\hline Base-collector saturation current & $I S C$ & $7.3 \times 10^{-14} \mathrm{~A}$ & $6 \times 10^{-11} \mathrm{~A}$ \\
\hline Forward knee current & $I K F$ & $125 \mathrm{~mA}$ & $190 \mathrm{~mA}$ \\
\hline Reverse knee current & $I K R$ & 0 & $61 \mathrm{~mA}$ \\
\hline Maximum forward current gain & $B F$ & 4.5 & 2.5 \\
\hline Maximum reverse current gain & $B R$ & 1.2 & 2 \\
\hline Base-emitter built-in potential & $V J E$ & $0.88 \mathrm{~V}$ & $0.3 \mathrm{~V}$ \\
\hline Base-collector built-in potential & $0.3 \mathrm{~V}$ & $0.3 \mathrm{~V}$ \\
\hline Emitter-base junction capacitance & $V J C$ & $2000 \mathrm{pF}$ & $60 \mathrm{pF}$ \\
\hline Collector-base junction capacitance & $C J E$ & $54 \mathrm{pF}$ & $52 \mathrm{pF}$ \\
\hline Emitter-base capacitance gradient factor & $C J C$ & 0.34 & 0.5 \\
\hline Collector-base capacitance gradient factor & $M J E$ & 0.5 & 0.5 \\
\hline Ideal forward transit time & $M J C$ & $0.3 \mu \mathrm{s}$ & $6 \mu \mathrm{s}$ \\
\hline Ideal reverse transit time & $T F$ & $1.5 \mu \mathrm{s}$ & $5 \mu \mathrm{s}$ \\
\hline Forward current emission coefficient & $T R$ & 0.95 & 1 \\
\hline Reverse current emission coefficient & $N F$ & 1 & 1 \\
\hline Low-current base-emitter emission coefficient & $N R$ & 1.7 & 1.2 \\
\hline Low-current base-collector emission coefficient & $N E$ & 1 & 1.2 \\
\hline
\end{tabular}

\subsection{Simulation Results}

In this section, using the thyristor model parameters determined in the previous section, the static and dynamic characteristics of the thyristor under study are simulated.

In order to turn-ON the thyristor, the gate current $I_{G}$ must be increased to a certain value. This value is found to be about $2.45 \mathrm{~mA}$ from measurements. The $I-V$ characteristics in this case are a full thyristor curve having the conventional S-shape as shown in Fig. 10. Also, Fig. 11 shows the measured and simulated $I-V$ curves for the diode mode of operation of the thyristor for different anode ranges. The results show good agreement between the simulation and measurements which indicates the usefulness of the model used for static characteristics.

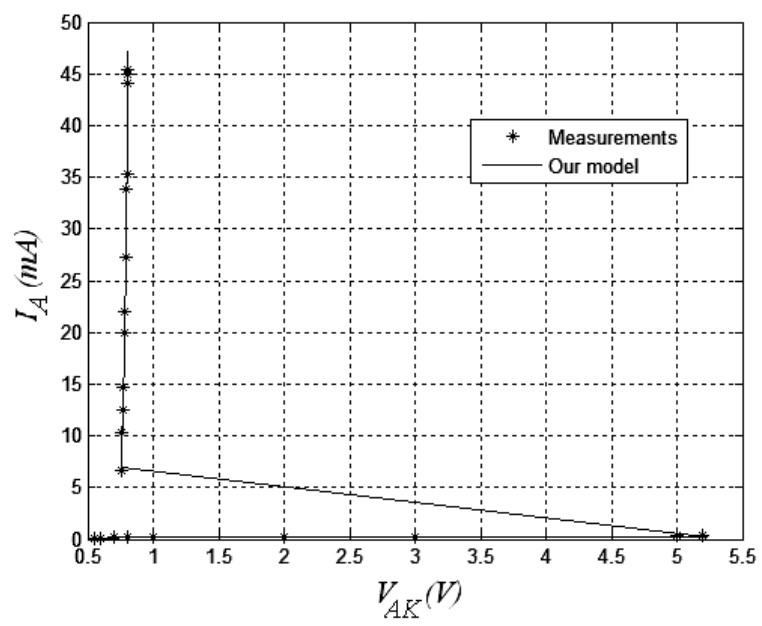

Fig 10 Measured and simulated I-V characteristics of the thyristor at the turn-ON point $\left(I_{G}=2.45 \mathrm{~mA}\right)$ (a)
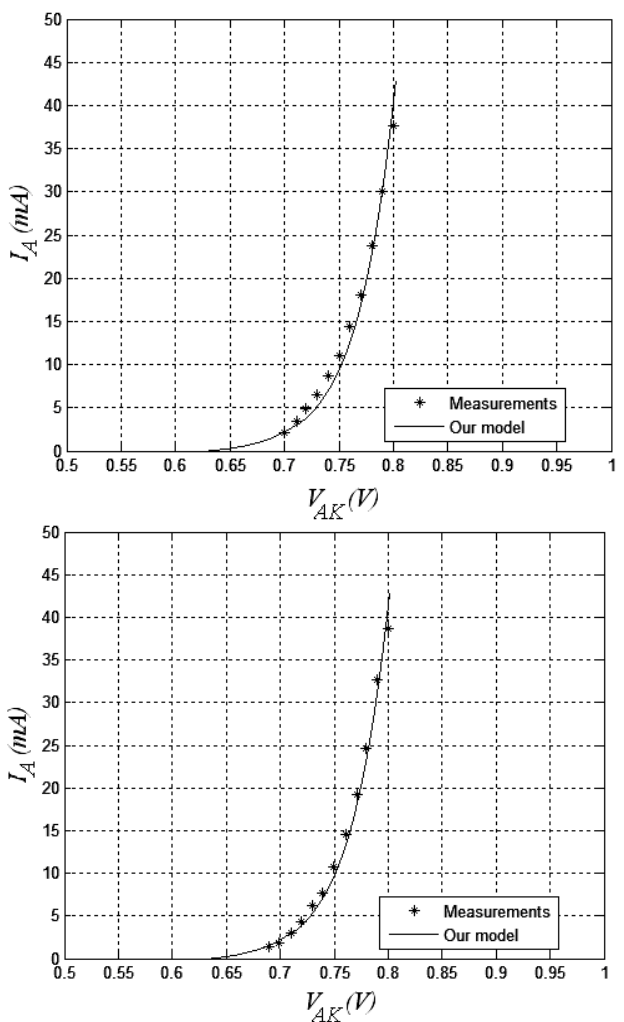

Fig 11 Measured and simulated I-V characteristics in the diode mode for (a) $I_{G}=8 \mathrm{~mA}$, and (b) $I_{G}=9.5 \mathrm{~mA}$

For the dynamic characteristics, the circuit shown in Fig. 12 is used for measurements and SPICE simulation. Fig. 13 shows the measured [9] and simulated switching waveforms at the same applied signals (square waveform of amplitude $=16 \mathrm{~V} \mathrm{p}$ $\mathrm{p}$, and frequency $=10 \mathrm{kHz}$ ). The waveforms are similar in shape and there is a good quantitative agreement between measured and simulated waveforms. This proves the validity of the model in tracing the dynamic behavior as well as the static one. 


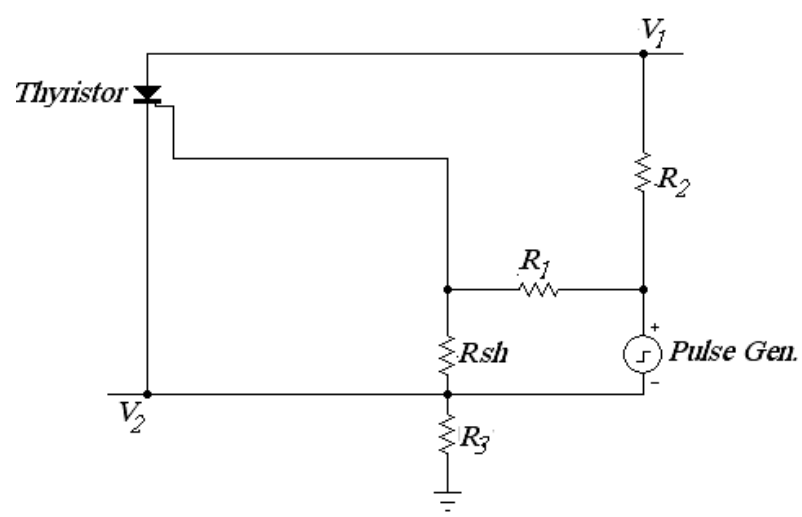

Fig 12 Circuit used for dynamic characteristics simulation
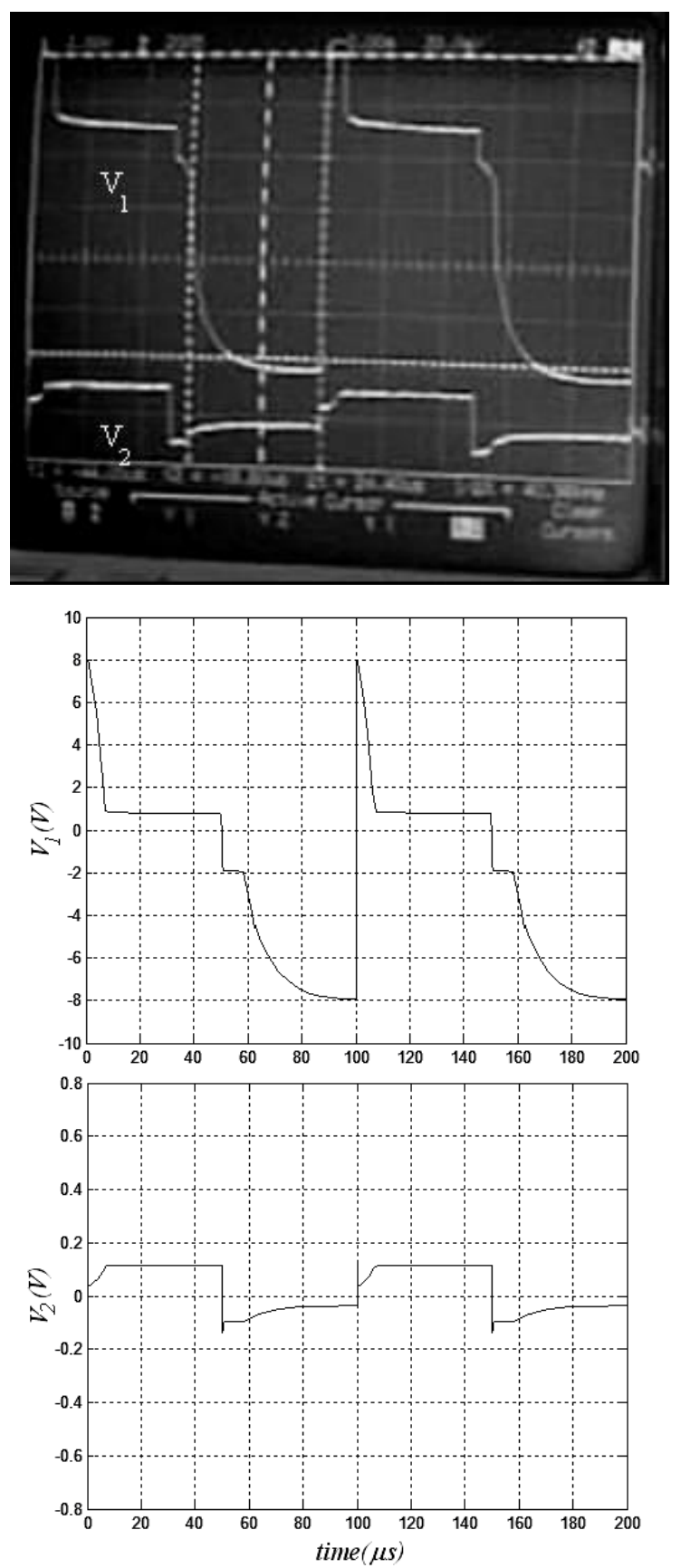

Fig 13 Measured and simulated switching waveforms of the anode to cathode voltage

$V_{\mathrm{AK}}=\mathrm{V}_{1}$ and the anode current $\mathrm{I}_{\mathrm{A}} \approx \mathrm{V}_{2} / \mathbf{R}_{\mathbf{3}}$

\section{CONCLUSIONS}

In this paper, an improved modeling approach for the power thyristor is presented. The model is based on the twotransistor configuration considering the Gummel-Poon model. It is demonstrated how to obtain the design parameters of the thyristor using simple analytical expressions. According to the design parameters, a SPICE parameters extraction using QUICKPIB simulator in Silvaco environment is accomplished. A case study was presented in details. The design and SPICE parameters were extracted. Static and dynamic behaviors were simulated using the SPICE model of the thyristor and compared to measurements. Very good agreement between simulations and measurements is observed showing the availability of the proposed model in tracing the various conditions of operation.

\section{REFERENCES}

[1] C. L. Ma, P. O. Lauritzen, P. Turkes, and H. J. Mattausch, "A physically-based lumped-charge SCR model,” In PESC 93 Rec., pp. 53-59, 1993.

[2] www.mathworks.com

[3] M. Kallala, "Représentation distribuée de la dynamique des charges dans la base large des thyristors Gate-Turnoff; application à un modèle de GTO pour la CA0 des circuits," Thèse de Doctorat de l'INSA de Toulouse, France, no d'ordre 317, 1994.

[4] http://www.tsr-be.com/logiciel/index.html

[5] B. W. Williams, "State-space thyristor computer model," Proc. Inst. Elect. Eng., Vol. 124, pp. 743-746, 1977.

[6] A. Brambilla and E. Dallago, "A circuit level simulation model of PNPN devices," IEEE Trans. Computer-Aided Design, Vol. 9. pp. 1254-1264, 1990.

[7] C. Hu and W. F. Ki, "Toward a practical computer-aid for thyristor circuit design," in PESC Rec., IEEE Power Electronics Specialists Conference, 1980, pp. 174-179.

[8] H. Xiangning, M. Li, and Q. Zhaoming, "A complete composite GTO model for circuit simulations," Journal of Electronics, vol. 15, no 2, pp. 130-137, 1998.

[9] G. T. Sayah, A. Zekry, H. F. Ragaie, and F. A. Soliman, "A SPICE model of a thyristor with high injection effects and conductivity modulation," Proc. of the 15th International Conf. on Microelectronics (ICM), 2003, pp. 344-347.

[10] G. Sayah, "Modeling and simulation of large area electronic devices," M. Sc. Thesis, Ain Shmas University, Faculty of Engineering, Cairo, 2002.

[11] A. G. M. Strollo, "A New SPICE Model of Power P-I-N Diode Based on Asymptotic Waveform Evaluation," IEEE Trans. Power Electronics, Vol. 12, No. 1, pp. 1220, 1997.

[12] W. Gerlach, “Thyristoren,” Sringer-Verlag, 1981.

[13] M. Hatle, and J. Vobecky, "A new approach to the simulation of small-signal current gains of pnpn structures," IEEE Trans. Elect. Dev., Vol. 40. No. 10, 1993.

[14] A. Zekry, "Untersuchung der zündausbreitung an thyristoren mit epitaxierter steuerbasis," PHD thesis, TU Berlin, 1981. 
[15] W. Gerlach, "Power devices physics," PHD Notes, unpublished.

[16] SILVACO International, 2D Process Simulation Software, Santa Clara, CA: Silvaco International.

[17] SILVACO International, 2D Device Simulation Software, Santa Clara, CA: Silvaco International.

[18] H. Garrab et al., "On the extraction of PiN diode design parameters for validation of integrated power converter design," IEEE Trans. On Power Electronics, Vol. 20, No. 3, pp. 660-670, 2005.

[19] P. D. Taylor, "Thyristor design and realization," chapter 3, New York: Wiley, 1993.

[20] B. J. Baliga, "Fundamentals of power semiconductor devices," chapter 8, New York: Springer, 2008.
[21] S. M. Sze, "Physics of Semiconductor Devices," chapter 2, New York: Wiley, 1981.

[22] F. Berz, "A simplified theory of the p-i-n diode," Solid State Electronics, Vol. 20, Issue 8, 1977, pp. 709-714.

[23] X. Kang, A. Caiafa, E. Santi, J.L. Hudgins, and P.R. Palmer, "Parameter extraction for a power diode circuit simulator model including temperature dependent effects," IEEE APEC Conf. Rec., pp. 452-458, Dallas, March 2002.

[24] SILVACO International, 1D Gummel_Poon SPICE Parameter Extraction Tool, Santa Clara, CA: Silvaco International. 